\title{
Graphene hybrids and extended defects: Revealing 3D structures and new insights to radiation damage
}

\author{
C. Hofer ${ }^{1}$, K. Mustonen ${ }^{1}$, A. Mittelberger ${ }^{1}$, M.R.A. Monazam ${ }^{1}$, A. Hussain², C. Mangler ${ }^{1}$, C. \\ Kramberger $^{1}$, E. I. Kauppinen ${ }^{2}$, T. Susi ${ }^{1}$, J. Kotakoski ${ }^{1}$, J. C. Meyer ${ }^{1}$ \\ 1. University of Vienna, Faculty of Physics, 1090 Vienna, Austria \\ ${ }^{2}$ Aalto University School of Science, Department of Applied Physics, FI-00076 Aalto, Finland
}

This presentation contains two different aspects of (scanning) transmission electron microscopy which were studied for extended defects in graphene and hybrids of graphene with other materials. The first topic is the extraction of 3D structural information from (in first approximation) projections of the structure. The second topic is radiation damage in organic molecular structures, where we obtained direct insight into the bond scission at low electron doses from atomic resolution imaging of a monolayer of chlorinated copper phtalocyanine $(\mathrm{ClCuPc})$ molecules on graphene.

Atomic resolution tomography is now developed to the point that the full 3D structure of small particles can be obtained without prior assumptions on their structure or chemistry [1]. However, this is implicitly based on the assumption that the sample does not change its structure during data acquisition. An alternative approach is a model-based quantitative analysis of the images, where a varying amount of reasonable assumptions - e.g. the presence of specific elements, or crystallinity - together with a statistical parameter estimation leads to a 3D model [2]. For analyzing 2D materials as well as ultra-thin free-standing heterostructures, a combination of a minimalistic tomographic approach with quantitative model-matching appears as a potential route to obtain 3D information with a minimum dose while at the same time not relying too much on prior assumptions. For extended defects in graphene (grain boundaries and small disordered patches), we used only two projections of the structure as input, and obtained a 3D model by an automated optimization where a minimum in the difference between simulated images of an atomistic model and the experimental images is obtained. Fig. 1a shows the example for a grain boundary in graphene [3]. It must be emphasized that not a single atom has changed its configuration between the two exposures. - however, much larger numbers of exposures (e.g. for a complete tomographic series) would be prohibited by the limited radiation stability of the sample. Fig. $1 \mathrm{~b}$ shows a single-walled carbon nanotube deposited on graphene [4]. As revealed by the 3D reconstruction, the graphene sheet is strongly deformed by the interaction with the nanotube.

For a hybrid system of $\mathrm{ClCuPc}$ molecules which form a 2D molecular crystal on graphene, we studied the effects of electron irradiation damage by using atomically resolved images with doses ranging from 100 to $100,000 \mathrm{e}^{-} / \AA^{2}$ [5]. From low dose exposures (Fig. 2a), high signal to noise images are generated by averaging over the periodic structure, revealing undamaged molecules (Fig. 2b). At higher doses, the chlorine atom is dissociated and the remaining fragments of the molecules form a cross-linked random network, which then remains stable under further irradiation (Fig. 2c). Importantly, the fragments of the molecules can still be identified by the presence of the heavy copper atom in the center (bright contrast), which enables a straightforward analysis of how the ionization damage and beam-induced cross linking proceeds.

[1] J. Miao et al., Science 353, p. 2157 (2016)

[2] S. Van Aert et al., IUCrJ, 3, 71-83 (2016) 
[3] C. Hofer et al., submitted

[4] K. Mustonen et al., in preparation

[5] A. Mittelberger, C. Kramberger, J. C. Meyer, https://arxiv.org/abs/1801.10409

[6] The authors acknowledge funding from the European Research Council (ERC) projects PICOMAT and ATMEN, the Austrian Science Fund (FWF) projects P25721-N20, P 28322-N36, and I3181-N36, and the Wiener Wissenschafts- Forschungs- und Technologiefonds (WWTF) via project MA14-009.
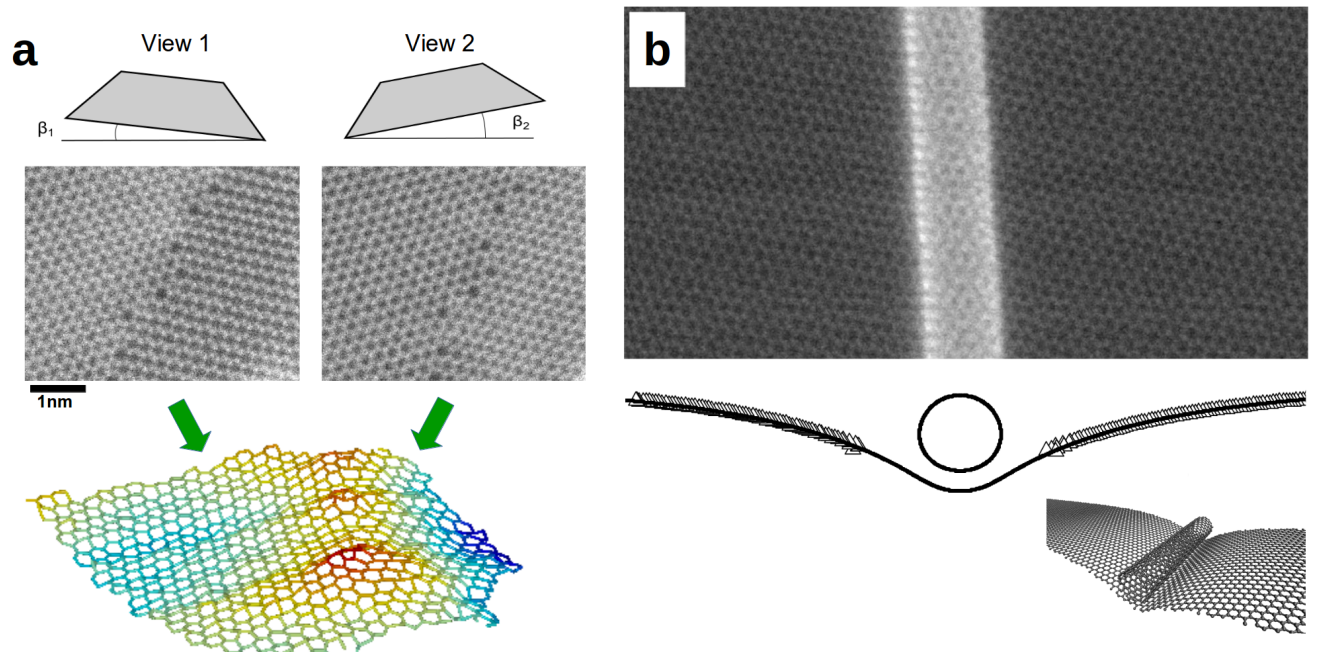

Figure 1. (a) 3D structure of a graphene grain boundary, obtained from two atomically resolved images with different view angles. (b) Image of a single-walled carbon nanotube on a graphene sheet, and 3D reconstruction of the graphene sheet in the vicinity of the nanotube. Triangles show experimental data averaged along the direction of the tube axis, while the solid line shows the results of a simulation. The inset shows a $3 \mathrm{D}$ view of the simulated structure.
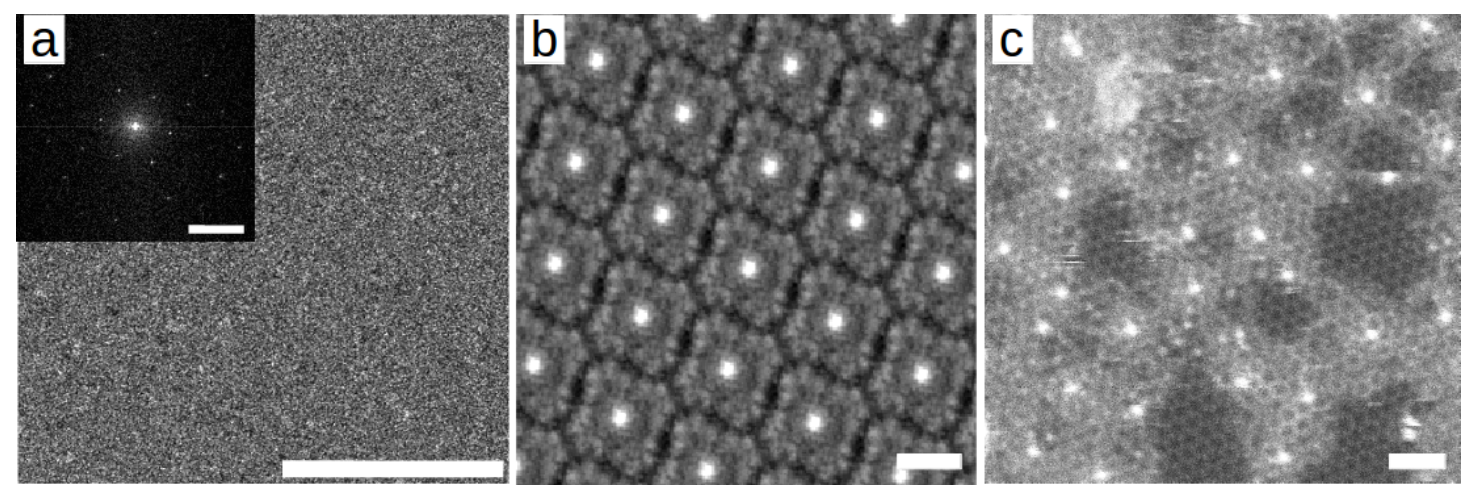

Figure 2. (a) Low-dose exposure of a ClCuPc monolayer on graphene $\left(144 \mathrm{e}^{-/} \AA^{2}\right)$. Inset shows a Fourier transform, displaying peaks of the 2D molecular crystal. (b) Image of the $\mathrm{ClCuPc}$ molecular layer obtained by periodic averaging of the low-dose exposure. (c) High-dose image showing crosslinked fragments of the molecules. Scale bars are $50 \mathrm{~nm}(\mathrm{a}), 1 \mathrm{~nm}^{-1}(\mathrm{a}$ inset), $1 \mathrm{~nm}(\mathrm{~b}+\mathrm{c})$. 